\title{
Cellular mechanisms of insulin resistance
}

\author{
Gerald I. Shulman \\ Howard Hughes Medical Institute, Departments of Internal Medicine and Cellular and Molecular Physiology, \\ Yale University School of Medicine, New Haven, Connecticut, USA \\ Address correspondence to: Gerald I. Shulman, Howard Hughes Medical Institute, Yale University School of \\ Medicine, 254C Boyer Center for Molecular Medicine, 295 Congress Avenue, New Haven, Connecticut 06510, \\ USA. Phone: (203) 785-5447; Fax: (203) 737-4059; E-mail: gerald.shulman@yale.edu.
}

It is estimated that by the year 2020 there will be approximately 250 million people affected by type 2 diabetes mellitus worldwide (1). Although the primary factors causing this disease are unknown, it is clear that insulin resistance plays a major role in its development. Evidence for this comes from (a) the presence of insulin resistance $10-20$ years before the onset of the disease $(2,3)$; (b) cross-sectional studies demonstrating that insulin resistance is a consistent finding in patients with type 2 diabetes (3-6); and (c) prospective studies demonstrating that insulin resistance is the best predictor of whether or not an individual will later become diabetic $(2,3)$. Here, I focus on some recent advances in our understanding of human insulin resistance that have been made using nuclear magnetic resonance spectroscopy (NMR). This technique takes advantage of the spin properties of the nuclei of certain isotopes, such as ${ }^{1} \mathrm{H},{ }^{13} \mathrm{C}$, and ${ }^{31} \mathrm{P}$, which endow the isotopes with a magnetic component that can be used to measure the concentration of intracellular metabolites noninvasively and to assess biochemical differences between normal and diabetic subjects. Drawing on NMR studies from my laboratory and others, I first consider the control of glucose phosphorylation and transport in regulating muscle responses to insulin. I then turn to the effects of fatty acids on insulin responses, showing that commonly accepted models that attempt to explain the association of insulin resistance and obesity are incompatible with recent findings. Finally, I propose an alternative model that appears to fit these and other available data.

\section{Contributions of muscle glycogen synthesis to} whole-body insulin-stimulated glucose metabolism Our initial studies addressed two questions. First, what is the contribution of insulin-stimulated muscle glycogen synthesis to whole-body insulin-stimulated glucose metabolism in normal individuals? Second, to what extent is this process defective in patients with type 2 diabetes (7)? We have measured rates of muscle glycogen synthesis using ${ }^{13} \mathrm{C}$ NMR spectroscopy to monitor the rate of $\left[1-{ }^{13} \mathrm{C}\right]$ glucose incorporation into muscle glycogen. Under steady-state plasma concentrations of insulin and glucose that mimic postprandial conditions, we found that muscle glycogen synthesis was approximate- ly $50 \%$ lower in diabetic subjects than in normal volunteers. When the mean rate of muscle glycogen synthesis was extrapolated to the whole body, the synthesis of muscle glycogen accounted for most of the whole-body glucose uptake, and virtually all of the nonoxidative glucose metabolism in both normal and diabetic subjects. These studies demonstrate that under hyperglycemic, hyperinsulinemic conditions, muscle glycogen synthesis is the major pathway for glucose metabolism in both normal and diabetic individuals, and that defective muscle glycogen synthesis plays a major role in causing insulin resistance in patients with type 2 diabetes.

The next major task is to identify the rate-controlling step in this process. Defects in glycogen synthase (8-10), hexokinase II (11-15), and glucose transport (14-17) have all been implicated in the loss of muscle glycogen synthesis in type 2 diabetics (Figure 1), making each of the corresponding biochemical events a potential target for antidiabetic therapy. To determine the relative importance of these steps to insulin-stimulated muscle glucose metabolism, we performed ${ }^{13} \mathrm{C}$ and ${ }^{31} \mathrm{P}$ NMR studies to measure intracellular concentrations of glucose, glucose-6-phosphate, and glycogen in muscle of patients with type 2 diabetes and muscle of age- and weight-matched control subjects (18). Intracellular glucose-6-phosphate is an intermediary metabolite between glucose transport and glycogen synthesis, so its intracellular concentration will respond to the relative activities of these two steps. In the event of decreased activity of glycogen synthase in diabetes, glucose-6-phosphate concentrations in diabetic patients would be expected to increase relative to that of normal individuals. Using ${ }^{31} \mathrm{P}$ NMR to assess intracellular glucose-6-phosphate concentrations under similar conditions of hyperglycemia and hyperinsulinemia as in the study discussed above, we found an increase of approximately $0.1 \mathrm{mM}$ in intracellular glucose-6-phosphate in normal individuals, and no change in patients with type 2 diabetes (18). The blunted incremental changes in glucose-6-phosphate in the type 2 diabetic patients in response to insulin stimulation can therefore be ascribed to either decreased glucose transport activity or decreased hexokinase II activity. 
To examine whether this defect in glucose transport or hexokinase II activity was a primary defect or an acquired defect secondary to other factors, such as glucose toxicity (19), we studied insulin-resistant offspring of parents with type 2 diabetes, examining the rate of muscle glycogen synthesis and the muscle glucose-6phosphate concentration under the same clamp conditions (20). Although these individuals were in all cases lean and normoglycemic, they were known to be at an approximately $40 \%$ increased risk for developing diabetes (2). Compared to age- and weight-matched control subjects, the children of diabetics had a $50 \%$ reduction in the rate of insulin-stimulated whole-body glucose metabolism, mainly due to a decrease in rates of muscle glycogen synthesis (20). Furthermore, their insulin-stimulated increment of intramuscular glucose-6-phosphate was severely reduced. This is consistent with impaired muscle glucose transport or reduced hexokinase II activity, and is similar to changes seen in patients with fully developed type 2 diabetes. When control subjects were studied at similar insulin levels but at euglycemia, both the rate of glycogen synthesis and the glucose-6-phosphate concentration decreased to values similar to that of the type 2 diabetic offspring. Therefore, we found that even before the onset of diabetes, insulin-resistant offspring of patients with type 2 diabetes have reduced rates of muscle glycogen synthesis that are secondary to a defect in either muscle glucose transport or hexokinase II activity. Clearly, defects in one or both of these activities occur early in the pathogenesis of type 2 diabetes.

To determine whether glucose transport or hexokinase II activity is rate controlling for insulin-stimulated muscle glycogen synthesis in patients with type 2 diabetes, we used a novel ${ }^{13} \mathrm{C}$ NMR method to assess intracellular glucose concentrations in muscle under similar hyperglycemic, hyperinsulinemic conditions as those used in the previous studies (21). Intracellular glucose is an intermediary metabolite between glucose transport and glucose phosphorylation, and its concentration reflects

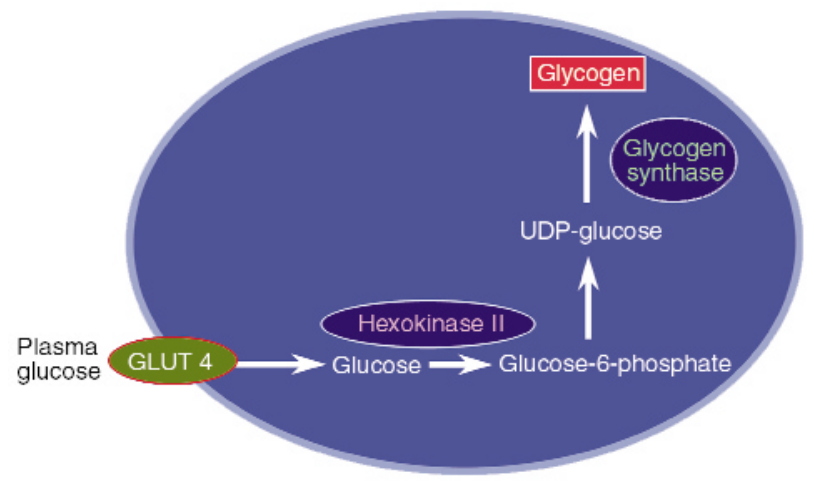

\section{Figure 1}

Potential rate-controlling steps responsible for reduced insulin-stimulated muscle glycogen synthesis in patients with type 2 diabetes mellitus. UDP, uridine diphosphate. the relative activities of glucose transporters (particularly GLUT4) and of hexokinase II. Unlike the standard biopsy method, this approach is noninvasive and is not subject to the errors caused by contamination of biopsy tissue with plasma glucose or incomplete removal of nonmuscle constituents. If hexokinase II activity is reduced relative to glucose transport activity in diabetes, one would predict a substantial increase in intracellular glucose (21), whereas if glucose transport is primarily responsible for maintaining intracellular glucose metabolism, intracellular glucose and glucose-6-phosphate should change proportionately. We found that the intracellular glucose concentration was far lower in the diabetic subjects than the concentration expected if hexokinase II was the primary rate-controlling enzyme for glycogen synthesis. When the rates of muscle glycogen synthesis in the diabetic subjects were increased by infusing greater amounts of insulin, the changes in the concentrations of intracellular glucose and glucose-6-phosphate indicated that the rates of glucose transport were matched by increases in the rates of glucose phosphorylation and glycogen synthesis. These data suggest a predominant role for glucose transport control of insulinstimulated muscle glycogen synthesis in patients with type 2 diabetes, but they do not rule out the possibility that there are other downstream abnormalities in the pathway of glycogen synthesis that do not exert rate-controlling effects under these conditions.

It has also been hypothesized that decreased delivery of substrate or insulin to the tissue bed might be responsible for the insulin resistance in type 2 diabetes (22). With regard to substrate delivery, we found no difference in the ${ }^{13} \mathrm{C} \mathrm{NMR}$-measured ratio of extra- to intracellular water space between the normal subjects and diabetic patients, implying that there were no significant differences between the groups that might be attributed to insulin-mediated vasodilatation. We also found no differences between the two groups in the interstitial insulin concentrations during the hyperinsulinemic clamps, suggesting that the delivery of insulin is not responsible for the insulin resistance in patients with type 2 diabetes. Overall, these data are consistent with the hypothesis that glucose transport is the rate-controlling step for insulin-stimulated muscle glycogen synthesis in patients with type 2 diabetes (see Pessin and Saltiel, this Perspective series, ref. 23), and that muscle glucose transport represents an important therapeutic target for this disease. These results also suggest that agents that enhance hexokinase II or glycogen synthase activity will not be as effective in improving insulin sensitivity in skeletal muscle of patients with type 2 diabetes as those that enhance glucose transport activity.

\section{Fatty acid-induced insulin resistance}

Increased plasma free fatty acid concentrations are typically associated with many insulin-resistant states, including obesity and type 2 diabetes mellitus (24-27). 
In a cross-sectional study of young, normal-weight offspring of type 2 diabetic patients, we found an inverse relationship between fasting plasma fatty acid concentrations and insulin sensitivity, consistent with the hypothesis that altered fatty acid metabolism contributes to insulin resistance in patients with type 2 diabetes (28). Furthermore, recent studies measuring intramuscular triglyceride content by muscle biopsy (29) or intramyocellular triglyceride content by ${ }^{1} \mathrm{H}$ NMR (30-32) have shown an even stronger relationship between accumulation of intramyocellular triglyceride and insulin resistance. In a classic series of studies, Randle et al. demonstrated that fatty acids compete with glucose for substrate oxidation in isolated rat heart muscle and rat diaphragm muscle. They speculated that increased fat oxidation causes the insulin resistance associated with obesity (33-35). The mechanism they proposed to explain the insulin resistance was that an increase in fatty acids caused an increase in the intramitochondrial acetyl CoA/CoA and $\mathrm{NADH} / \mathrm{NAD}^{+}$ratios, with sub-

\section{Figure 2}

Top: Mechanism of fatty acid-induced insulin resistance in skeletal muscle as proposed by Randle et al. An increase in fatty acid concentration results in an elevation of the intramitochondrial acetyl $\mathrm{CoA} / \mathrm{CoA}$ and $\mathrm{NADH} / \mathrm{NAD}^{+}$ratios, with subsequent inactivation of pyruvate dehydrogenase. This in turn causes citrate concentrations to increase, leading to inhibition of phosphofructokinase. Subsequent increases in intracellular glucose-6-phosphate concentration would inhibit hexokinase II activity, which would result in an increase in intracellular glucose concentration and a decrease in muscle glucose uptake. Bottom: Proposed alternative mechanism for fatty acid-induced insulin resistance in human skeletal muscle. An increase in delivery of fatty acids to muscle or a decrease in intracellular metabolism of fatty acids leads to an increase in intracellular fatty acid metabolites such as diacylglycerol, fatty acyl CoA, and ceramides. These metabolites activate a serine/threonine kinase cascade (possibly initiated by protein kinase $(\theta)$ leading to phosphorylation of serine/threonine sites on insulin receptor substrates (IRS-1 and IRS-2), which in turn reduces the ability of the insulin receptor substrates to activate PI 3-kinase. As a consequence, glucose transport activity and other events downstream of insulin receptor signaling are diminished. HK, hexokinase II; PFK, phosphofructokinase; PDH, pyruvate dehydrogenase; $\mathrm{PKC} \theta$, protein kinase $\mathrm{C} \theta$. sequent inactivation of pyruvate dehydrogenase (Figure 2, top). This in turn would cause intracellular citrate concentrations to increase, leading to inhibition of phosphofructokinase, a key rate-controlling enzyme in glycolysis. Subsequent accumulation of glucose-6phosphate would inhibit hexokinase II activity, resulting in an increase in intracellular glucose concentrations and decreased glucose uptake.

A recent series of studies by our group has challenged this conventional hypothesis (36-38). In the first study, we used ${ }^{13} \mathrm{C}$ and ${ }^{31} \mathrm{P}$ NMR spectroscopy to measure skeletal muscle glycogen and glucose-6-phosphate concentrations in healthy subjects. The subjects were maintained in euglycemic, hyperinsulinemic conditions with either low or high levels of plasma fatty acids (36). Increasing the plasma fatty acid concentration for 5 hours caused a reduction of approximately $50 \%$ in

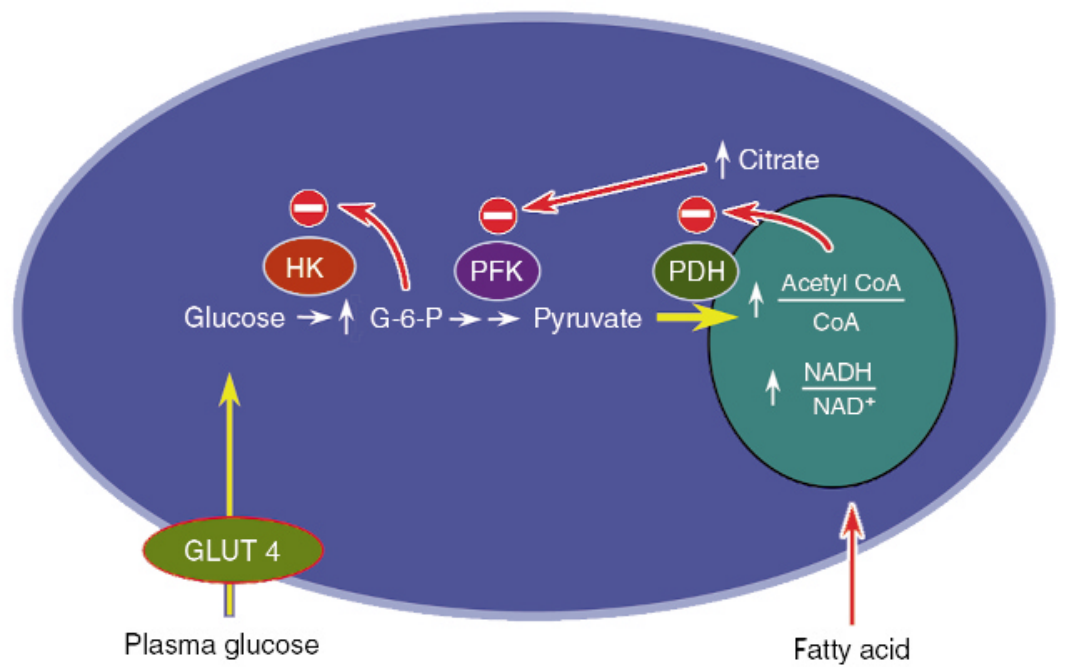

Insulin

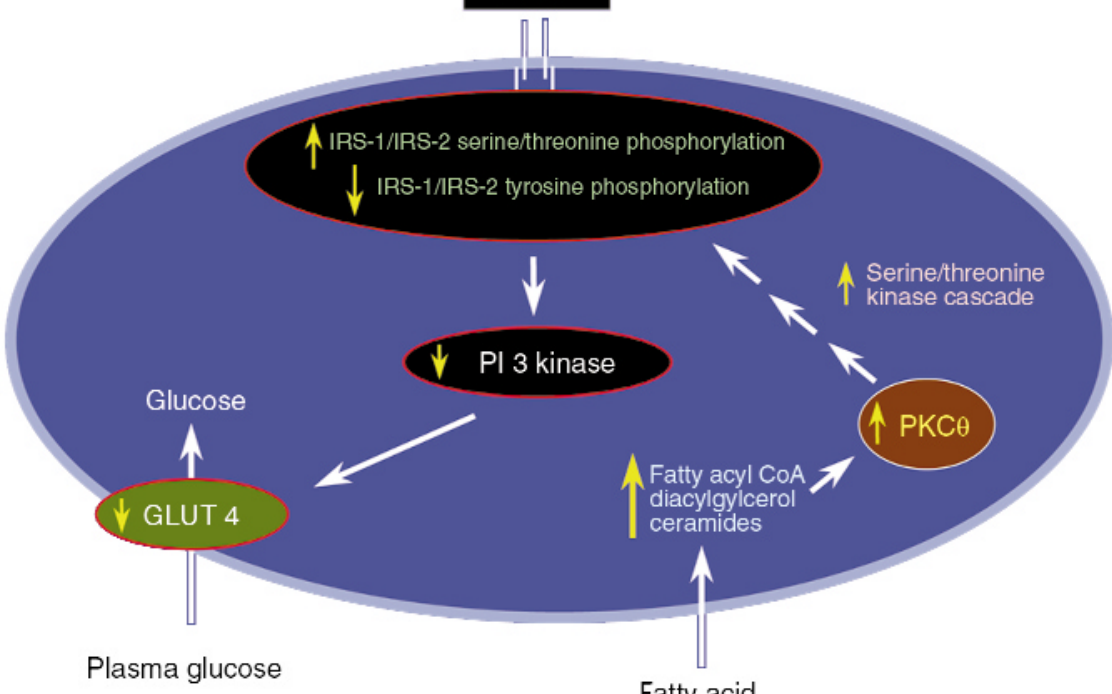


insulin-stimulated rates of muscle glycogen synthesis and whole-body glucose oxidation compared to controls. In contrast to the results from the model of Randle and coworkers, which predicted that fat-induced insulin resistance would result in an increase in intramuscular glucose-6-phosphate (34), we found that the drop in muscle glycogen synthesis was preceded by a fall in intramuscular glucose-6-phosphate. These data suggest that increases in plasma fatty acid concentrations initially induce insulin resistance by inhibiting glucose transport or phosphorylation activity, and that the reduction in muscle glycogen synthesis and glucose oxidation follows. The reduction in insulin-activated glucose transport and phosphorylation activity in normal subjects maintained at high plasma fatty acid levels is similar to that seen in obese individuals (39), patients with type 2 diabetes (18), and lean, normoglycemic insulin-resistant offspring of type 2 diabetic individuals (20). Hence, accumulation of intramuscular fatty acids (or fatty acid metabolites) appears to play an important role in the pathogenesis of insulin resistance seen in obese patients and patients with type 2 diabetes. Moreover, fatty acids seem to interfere with a very early step in insulin stimulation of GLUT4 transporter activity or hexokinase II activity. This conclusion is at odds with the mechanism proposed by Randle et al. (33-35), which predicts an increase in intramuscular glucose-6-phosphate concentrations resulting from inhibitory effects of fatty acid on phosphofructokinase activity (due to an increase in intracellular citrate concentration).

To distinguish between possible effects of fatty acids on glucose transport activity and on hexokinase II activity, we measured intracellular concentrations of glucose in muscle using ${ }^{13} \mathrm{C} \mathrm{NMR}$ (37). The logic of this experiment was similar to that described above, in which we followed concentrations of glucose-6-phosphate to determine the relative activity of glucose transport and glycogen synthesis. Because intracellular glucose is an intermediary metabolite between glucose transport and hexokinase II, its concentration reflects the relative activities of these two steps. If a decrease in hexokinase II activity was responsible for the lower rate of insulin-stimulated muscle glycogen synthesis, intracellular glucose concentrations should increase. However, if the impairment was at the level of glucose transport, there should be no difference or a decrease in the intracellular glucose concentration. We found that elevated plasma fatty acid concentrations caused a significant reduction in intracellular glucose concentration in the lipid infusion studies compared to control studies in which glycerol (the other metabolite released by lipolysis) was infused in the absence of any exogenous fatty acid. These data imply that the rate-controlling step for fatty acid-induced insulin resistance in humans is glucose transport, and offer further evidence against the Randle mechanism, which predicts an increase in both intracellular glucose-6-phosphate and glucose concentrations.

This reduced glucose transport activity could be the result of fatty acid effects on the GLUT4 transporter directly - alterations in the trafficking, budding, fusion, or activity of GLUT4 (40) - or it could result from fatty acid-induced alterations in upstream insulin signaling events, resulting in decreased GLUT4 translocation to the plasma membrane (Figure 2, bottom). To explore the latter possibility, we examined IRS-1-associated phosphatidylinositol 3-kinase (PI 3kinase) activity in muscle biopsy samples, using the identical lipid infusion protocol described above for study of fatty acid effects. We found that elevations in plasma fatty acid concentrations similar to the previous NMR studies $(36,37)$ abolished insulin-stimulated, IRS-1-associated PI 3-kinase activity compared with a fourfold insulin stimulation observed in the glycerolcontrol infusion studies (37). The reduced insulinstimulated PI 3-kinase activity may be due to a direct effect of intracellular free fatty acids (or some fatty acid metabolite) on PI 3-kinase, or may be secondary to alterations in upstream insulin signaling events. Consistent with an indirect effect, we found that a similar lipid infusion protocol in rats resulted in a reduction of insulin-stimulated IRS-1 tyrosine phosphorylation, which was associated with activation of protein kinase C $\theta$ (38), a known serine kinase that has been shown to be activated by diacylglycerol (41). High-fat feeding has also been shown to both increase the content of longchain fatty acyl CoA in muscle and alter the protein kinase $\mathrm{C}$ isoenzymes $\theta$ and $\varepsilon$ (42).

\section{A unifying hypothesis for common forms of human insulin resistance}

An attractive hypothesis to account for the effects of fatty acids in muscle cells is shown in Figure 2, bottom panel. This model holds that increasing intracellular fatty acid metabolites, such as diacylglycerol, fatty acyl CoA's, or ceramides activates a serine/threonine kinase cascade (possibly initiated by protein kinase $\mathrm{C} \theta$ ), leading to phosphorylation of serine/threonine sites on insulin receptor substrates. Serine-phosphorylated forms of these proteins fail to associate with or to activate PI 3-kinase, resulting in decreased activation of glucose transport and other downstream events. If this hypothesis is correct, any perturbation that results in accumulation of intracellular fatty acyl CoA's or other fatty acid metabolites in muscle and liver, either through increased delivery or decreased metabolism, might be expected to induce insulin resistance. Evidence supporting this hypothesis comes from recent studies in transgenic mice that are almost totally devoid of fat because their adipocytes express the AZIP/F-1 protein, which blocks the function of several classes of transcription factors (43). These mice are severely insulin resistant, due to defects in insulin 
action, particularly IRS-1/IRS-2-dependent activation of PI 3-kinase, in muscle and liver (44). Interestingly, these abnormalities were associated with a twofold increase in muscle and liver triglyceride content, and upon transplantation of fat tissue into these mice, triglyceride content in muscle and liver returned to nor$\mathrm{mal}$, as did insulin signaling and action. These findings are consistent with the hypothesis that insulin resistance develops in obesity, type 2 diabetes, and lipodystrophy because of alterations in the partitioning of fat between the adipocyte and muscle or liver. This change leads to the intracellular accumulation of triglycerides, and, probably more importantly, of intracellular fatty acid metabolites (fatty acyl CoA's, diacylglycerol, and ceramides, among others) in these insulin-responsive tissues, which leads to acquired insulin signaling defects and insulin resistance (Figure 2, bottom).

This hypothesis might also explain how thiazolidinediones improve insulin sensitivity in muscle and liver tissue. By activating PPAR- $\gamma$ receptors in adipocytes and promoting adipocyte differentiation, these agents might promote a redistribution of fat from liver and muscle into the adipocytes, much as fat transplantation does in fat-deficient mice (44). This hypothesis is supported by some recent thiazolidinedione studies in rats fed high-fat diets $(45,46)$. It might also be expected that any alteration in the ability of muscle and liver to metabolize fatty acids, such as inherited or acquired defects in mitochondria function, would also lead to intracellular accumulation of fatty acid metabolites and subsequent defects in insulin signaling and action. Given the polygenic nature of type 2 diabetes, it is likely that examples of both of these possibilities will be identified. This mechanism, if it proves to be correct, offers many new therapeutic targets for novel insulin-sensitizing agents.

\section{Acknowledgments}

Space limitations preclude this from being a comprehensive review, and this unfortunately limits appropriate recognition of many of my colleagues worldwide who have contributed immeasurably to the development of this field. However, I specifically wish to thank Kitt Petersen, Gary Cline, and Douglas Rothman for their scientific contributions, advice, and editorial assistance, and Dennis McGarry for many stimulating discussions. The studies summarized in this review were supported in part by grants from the NIH (R01 DK-49230, R01 DK-40936, and P30 DK-45735). G.I. Shulman is an investigator of the Howard Hughes Medical Institute.

1. O'Rahilly, S. 1997. Science, medicine, and the future. Non-insulin dependent diabetes mellitus: the gathering storm. BMJ. 314:955-959.

2. Warram, J.H., Martin, B.C., Krolewski, A.S., Soeldner, J.S., and Kahn, C.R. 1990. Slow glucose removal rate and hyperinsulinemia precede the development of type II diabetes in the offspring of diabetic patients. Ann. Intern. Med. 113:909-915.
3. Lillioja, S., et al. 1988. Impaired glucose tolerance as a disorder of insulin action. Longitudinal and cross-sectional studies in Pima Indians. N. Engl. J. Med. 318:1217-1225.

4. Haffner, S.M., et al. 1990. Diminished insulin sensitivity and increased insulin response in nonobese, nondiabetic Mexican Americans. Metabolism. 39:842-847.

5. Reaven, G.M., Bernstein, R., Davis, B., and Olefsky, J.M. 1976. Nonketotic diabetes mellitus: insulin deficiency or insulin resistance? Am.J. Med. 60:80-88.

6. DeFronzo, R.A. 1988. The triumvirate: beta-cell, muscle, liver: a collusion responsible for NIDDM. Diabetes. 37:667-687.

7. Shulman, G.I., et al. 1990. Quantitation of muscle glycogen synthesis in normal subjects and subjects with non-insulin-dependent diabetes by ${ }^{13} \mathrm{C}$ nuclear magnetic resonance spectroscopy. N. Engl. J. Med. 322:223-228.

8. Bogardus, C., Lillioja, S., Stone, K., and Mott, D. 1984. Correlation between muscle glycogen synthase activity and in vivo insulin action in man. J. Clin. Invest. 73:1185-1190.

9. Damsbo, P., Vaag, A., Hother-Nielsen, O., and Beck-Nielsen, H. 1991. Reduced glycogen synthase activity in skeletal muscle from obese patients with and without type 2 (non-insulin-dependent) diabetes mellitus. Diabetologia. 34:239-245.

10. Wright, K.S., Beck, N.H., Kolterman, O.G., and Mandarino, L.J. 1988. Decreased activation of skeletal muscle glycogen synthase by mixed-meal ingestion in NIDDM. Diabetes. 37:436-440.

11. Kelley, D.E., et al. 1996. The effect of non-insulin-dependent diabetes mellitus and obesity on glucose transport and phosphorylation in skeletal muscle. J. Clin. Invest. 97:2705-2713.

12. Braithwaite, S.S., Palazuk, B., Colca, J.R., Edwards, C.W., III, and Hofmann, C. 1995. Reduced expression of hexokinase II in insulin-resistant diabetes. Diabetes. 44:43-48.

13. Kruszynska, Y.T., Mulford, M.I., Baloga, J., Yu, J.G., and Olefsky, J.M. 1998. Regulation of skeletal muscle hexokinase II by insulin in nondiabetic and NIDDM subjects. Diabetes. 47:1107-1113.

14. Rothman, D.L., Shulman, R.G., and Shulman, G.I. 1992. 31P nuclear magnetic resonance measurements of muscle glucose-6-phosphate. Evidence for reduced insulin-dependent muscle glucose transport or phosphorylation activity in non-insulin-dependent diabetes mellitus. J. Clin. Invest. 89:1069-1075.

15. Bonadonna, R.C., et al. 1996. Roles of glucose transport and glucose phophorylation in muscle insulin resistance of NIDDM. Diabetes. 45:915-925.

16. Zierath, J.R., et al. 1996. Insulin action on glucose transport and plasma membrane GLUT4 content in skeletal muscle from patients with NIDDM. Diabetologia. 39:1180-1189.

17. Dohm, G.L., et al. 1988. An in vitro human muscle preparation suitable for metabolic studies. Decreased insulin stimulation of glucose transport in muscle from morbidly obese and diabetic subjects. J. Clin. Invest. 82:486-494.

18. Rothman, D.L., Shulman, R.G., and Shulman, G.I. 1992. ${ }^{31} \mathrm{P}$ nuclear magnetic resonance measurements of muscle glucose-6-phosphate: evidence for reduced insulin-dependent muscle glucose transport or phosphorylation activity in non-insulin-dependent diabetes mellitus. J. Clin. Invest. 89:1069-1075.

19. Rossetti, L., Giaccari, A., and DeFronzo, R.A. 1990. Glucose toxicity. Diabetes Care. 13:610-630.

20. Rothman, D.L., et al. 1995. Decreased muscle glucose transport/phosphorylation is an early defect in the pathogenesis of non-insulindependent diabetes mellitus. Proc. Natl. Acad. Sci. USA. 92:983-987.

21. Cline, G., et al. 1999. Glucose transport is rate controlling for insulin stimulated muscle glycogen synthesis in type 2 diabetes. N. Engl. J. Med. 341:240-246.

22. Yang, Y.J., Hope, I.D., Ader, M., and Bergman, R.N. 1989. Insulin transport across capillaries is rate limiting for insulin action in dogs. J. Clin. Invest. 84:1620-1628.

23. Pessin, J.E., and Saltiel, A.R. 2000. Signaling pathways in insulin action: molecular targets of insulin resistance. J. Clin. Invest. 106:165-169.

24. Reaven, G.M., Hollenbeck, C., Jeng, C.-Y., Wu, M.S., and Chen, Y.-D. 1988. Measurement of plasma glucose, free fatty acid, lactate, and insulin for $24 \mathrm{~h}$ in patients with NIDDM. Diabetes. 37:1020-1024.

25. Frayne, K.N. 1993. Insulin resistance and lipid metabolism. Curr. Opin. Lipidol. 4:197-204.

26. McGarry, J.D. 1992. What if Minkowski had been ageusic? An alternative angle on diabetes. Science. 258:766-770.

27. Boden, G., Chen, X., Ruiz, J., White, J.V., and Rosetti, L. 1994. Mechanism of fatty acid induced inhibition of glucose uptake. J. Clin. Invest. 
93:2438-2446.

28. Perseghin, G., Ghosh, S., Gerow, K., and Shulman, G.I. 1997. Metabolic defects in lean nondiabetic offspring of NIDDM parents: a cross-sectional study. Diabetes. 46:1001-1009.

29. Pan, D.A., et al. 1997. Skeletal muscle triglyceride levels are inversely related to insulin action. Diabetes. 46:983-988.

30. Krssak, M., et al. 1999. Intramyocellular lipid concentrations are correlated with insulin sensitivity in humans: a ${ }^{1} \mathrm{H}$ NMR spectroscopy study. Diabetologia. 42:113-116.

31. Perseghin, G., et al. 1999. Intramyocellular triglyceride content is a determinant of in vivo insulin resistance in humans: a $1 \mathrm{H}-13 \mathrm{C}$ nuclear magnetic resonance spectroscopy assessment in offspring of type 2 diabetic parents. Diabetes. 48:1600-1606.

32. Stein, D.T., Szczepaniak, L.S., Dobbins, R.L., Snell, P., and McGarry, J.D. 1998. Skeletal muscle triglycerides stores are increased in insulin resistant states. Proceedings of the 6 th Scientific Meeting for the International Society for Magnetic Resonance in Medicine (Sydney). 388. (Abstr.).

33. Randle, P.J., Garland, P.B., Hales, C.N., and Newsholme, E.A. 1963. The glucose fatty-acid cycle: its role in insulin sensitivity and the metabolic disturbances of diabetes mellitus. Lancet. i:785-789.

34. Randle, P.J., Garland, P.B., Newsholme, E.A., and Hales, C.N. 1965. The glucose fatty-acid cycle in obesity and maturity onset diabetes mellitus. Ann. NY Acad. Sci. 131:324-333.

35. Randle, P.J., Newsholme, E.A., and Garland, P.B. 1964. Regulation of glucose uptake by muscle. Effects of fatty acids, ketone bodies and pyruvate, and of alloxan, diabetes and starvation, on the uptake and metabolic fate of glucose in rat heart and diaphragm muscles. Biochem. J. 93:652-665.

36. Roden, M., et al. 1996. Mechanism of free fatty acid-induced insulin resistance in humans. J. Clin. Invest. 97:2859-2865.
37. Dresner, A., et al. 1999. Effects of free fatty acids on glucose transport and IRS-1-associated phosphatidylinositol 3-kinase activity. J. Clin. Invest. 103:253-259.

38. Griffin, M.E., et al. 1999. Free fatty acid-induced insulin resistance is associated with activity of protein kinase $C$ theta and alterations in the insulin signaling cascade. Diabetes. 48:1270-1274.

39. Petersen, K.F., et al. 1998. 13C/31P NMR studies on the mechanism of insulin resistance in obesity. Diabetes. 47:381-386.

40. Kahn, B.B. 1992. Facilitative glucose transporters: regulatory mechanisms and dysregulation in diabetes. J. Clin. Invest. 89:1367-1374.

41. Chalkley, S.M., Hettiarachchi, N., Chisholm, D.J., and Kraegen, E.W. 1998. Five-hour fatty acid elevation increases muscle lipids and impairs glycogen synthesis in the rat. Metabolism. 47:1121-1126.

42. Schmitz-Peiffer, C., et al. 1997. Alterations in the expression and cellular localization of protein kinase $\mathrm{C}$ isozymes epsilon and theta are associated with insulin resistance in skeletal muscle of the high-fat-fed rat. Diabetes. 46:169-178.

43. Gavrilova, O., et al. 2000. Surgical implantation of adipose tissue reverses diabetes in lipoatrophic mice. J. Clin. Invest. 105:271-278.

44. Kim, J., Gavrilova, O., Chen, Y., Reitman, M., Shulman, G.I. 2000. Mechanisms of insulin resistance in A-ZIP/F-1 fatless mice. J. Biol. Chem. 275:8456-8460.

45. Oakes, N.D., et al. 1994. A new antidiabetic agent, BRL 49653, reduces lipid availability and improves insulin action and glucoregulation in the rat. Diabetes. 43:1203-1210.

46. Oakes, N.D., Camilleri, S., Furler, S.M., Chisholm, D.J., and Kraegen, E.W. 1997. The insulin sensitizer, BRL 49653, reduces systemic fatty acid supply and utilization and tissue lipid availability in the rat. Metabolism. 46:935-942. 